\title{
Ocular Pharmacological Profile of Hydrocortisone in Dry Eye Disease
}

\section{OPEN ACCESS}

Edited by:

Benedetto Falsini,

Catholic University of the Sacred

Heart, Italy

Reviewed by:

Rosario Amato,

University of Miami Health System,

United States

Tomo Suzuki,

Kyoto Prefectural University of

Medicine, Japan

${ }^{*}$ Correspondence:

Claudio Bucolo

claudio.bucolo@unict.it

Specialty section: This article was submitted to

Experimental Pharmacology

and Drug Discovery,

a section of the journal

Frontiers in Pharmacology

Received: 24 July 2019 Accepted: 27 September 2019

Published: 18 October 2019

Citation:

Bucolo C, Fidilio A, Fresta CG,

Lazzara F, Platania CBM,

Cantarella G, Di Benedetto G, Burgaletto C, Bernardini R, Piazza C,

Barabino S and Drago F (2019)

Ocular Pharmacological Profile of Hydrocortisone in Dry Eye Disease.

Front. Pharmacol. 10:1240.

doi: 10.3389/fphar.2019.01240

\begin{abstract}
Claudio Bucolo ${ }^{1,2 *}$, Annamaria Fidilio ${ }^{1}$, Claudia Giuseppina Fresta ${ }^{1}$, Francesca Lazzara ${ }^{1}$, Chiara Bianca Maria Platania', Giuseppina Cantarella', Giulia Di Benedetto', Chiara Burgaletto ${ }^{1}$, Renato Bernardini ${ }^{1}$, Cateno Piazza ${ }^{3}$, Stefano Barabino ${ }^{4}$ and Filippo Drago ${ }^{1,2}$

${ }^{1}$ Department of Biomedical and Biotechnological Sciences, School of Medicine, University of Catania, Catania, Italy, ${ }^{2}$ Center for Research in Ocular Pharmacology-CERFO, University of Catania, Catania, Italy, ${ }^{3}$ Analytical Department, University of Catania Consortium Unifarm, Catania, Italy, ${ }^{4}$ Ocular Surface and Dry Eye Center, Ospedale L. Sacco, University of Milano, Milano, Italy
\end{abstract}

To investigate the ocular pharmacological profile of hydrocortisone $(\mathrm{HC})$ using in vitro and in vivo models of dry eye disease. Rabbit corneal epithelial cells (SIRCs) were used to assess the effect of $\mathrm{HC}$ in two paradigms of corneal damage: hyperosmotic stress and scratch-wound assay. Dry eye was induced in albino rabbits by topical administration of atropine sulfate or by injection of concanavalin A (ConA) into the lacrimal gland. TNF $\alpha$, TNF-related apoptosis-inducing ligand (TRAIL), IL-1 $\beta$, and IL-8 were determined by ELISA or western blot in a corneal damage hyperosmotic in vitro model, with or without $\mathrm{HC}$ treatment. Inflammatory biomarkers, such as TNF $\alpha$, IL-8, and MMP-9, were evaluated in tears of rabbit eye injected with ConA and treated with $\mathrm{HC}$. Tear volume and tear film integrity, in both in vivo models, were evaluated by the Schirmer test and tear break-up time (TBUT). Ocular distribution of four formulations containing HC $(0.001 \%, 0.003 \%$, $0.005 \%$, and $0.33 \%$ ) was performed in the rabbit eye. Aqueous humor samples were collected after 15, 30, 60, and 90 min from instillation and then detected by LC-MS/MS. Hyperosmotic insult significantly activated protein expression of inflammatory biomarkers, which were significantly modulated by $\mathrm{HC}$ treatment. $\mathrm{HC}$ significantly enhanced the re-epithelialization of scratched SIRCs. Treatment with $\mathrm{HC}$ eye drops significantly reduced the tear concentrations of TNF- $\alpha, \mathrm{IL}-8$, and MMP-9 vs. vehicle in the ConA dry eye model. Moreover, $\mathrm{HC}$ significantly restored the tear volume and tear film integrity to levels of the control eyes, both in ConA- and atropine-induced dry eye paradigms. Finally, we demonstrated that $\mathrm{HC}$ crossed, in a dose-dependent manner, the corneal barrier when the eyes were topically treated with $\mathrm{HC}$ formulations (dose range $0.003-0.33 \%$ ). No trace of $\mathrm{HC}$ was detected in the aqueous humor after ocular administration of eye drops containing the lowest dose of the drug $(0.001 \%)$, indicating that, at this very low concentration, the drug did not pass the corneal barrier avoiding potential side effects such as intraocular pressure rise. Altogether, these data suggest that $\mathrm{HC}$, at very low concentrations, has an important anti-inflammatory effect both in vitro and in vivo dry eye paradigms and a good safety profile.

Keywords: dry eye disease, inflammation, cornea, hydrocortisone, Sjögren syndrome 


\section{INTRODUCTION}

Corticosteroids are potent anti-inflammatory drugs that exert their pharmacological effects by binding to the glucocorticoid receptor (GR), leading to the regulation of gene expression by transrepression (i.e., regulating transcriptional regulators such as NF-kB) (Bekhbat et al., 2017). Ophthalmic eye drops of corticosteroids such as dexamethasone, triamcinolone, fluocinolone, loteprednol, and prednisolone are generally used in clinical practice to manage several ocular conditions, such as dry eye disease (DED) (Pinto-Fraga et al., 2016), conjunctivitis (Ridolo et al., 2014), uveitis, diabetic macular edema (Caceresdel-Carpio et al., 2016), and postsurgery inflammation (Aptel et al., 2017; Wielders et al., 2018). Dry eye has a disparate etiology that includes, among others, endocrine, immune, and iatrogenic factors, which can modify tear film homeostasis leading to inflammation (Musumeci et al., 2008; Bucolo et al., 2011; Lessard et al., 2013; Moutsopoulos, 2014; Bron et al., 2017). DED was originally considered a disease elicited by a deficient volume and quality of the tears. The Dry Eye Workshop (DEWS) of the Tear Film Ocular Surface Society (TFOS) described DED as "a multifactorial disease of the tears and ocular surface that results in symptoms of discomfort, visual disturbance, and tear film instability with potential damage to the ocular surface. It is accompanied by increased osmolarity of the tear film and inflammation of the ocular surface" (Craig et al., 2017). The canonical approach to treat DED focuses on tear replacement with artificial tears or, alternatively, on preserving the patient's tears by means of occlusion of the tear drainage system (Ervin et al., 2017). These approaches have been demonstrated to decrease symptoms and signs of dry eye (Pucker et al., 2016), improving the resultant blurred vision and ocular discomfort (Liu and Pflugfelder, 1999). However, these treatments are considered palliative simply because they do not address the immunoinflammatory process, that underlies the disease. Although the trigger of the pathogenic mechanism that leads to DED is not currently known, a growing body of evidence suggests that DED is characterized by an inflammatory process, which affects the lacrimal gland-ocular surface functional unit (Stern and Pflugfelder, 2004). The inflammation process feeds a vicious cycle of tear deficiency, contributing to ocular surface damage that in turn leads to symptoms and signs of chronic dry eye. Although artificial tears can have an indirect anti-inflammatory effect by lowering tear osmolarity and diluting pro-inflammatory factors on the ocular surface, they are not able to completely block this inflammatory vicious cycle. Ocular surface inflammation is crucial in the pathophysiology of dry eye, therefore antiinflammatory therapy, including corticosteroids, may be of benefit to DED patients. Corticosteroids are prescription drugs for the treatment of DED along with antibiotics, integrin antagonists such as lifitegrast, and immunomodulatory drugs such as cyclosporine. In general, the use of prescription drugs for DED needs to be considered in the context of the single clinical history of the patient, along with the relative level of evidence supporting their use for that specific indication, as these drugs significantly differs in mechanism of action, and potential side effects. In spite of the remarkable anti-inflammatory properties of corticosteroids, chronic use often leads to undesired side effects, which are mainly related to the ability of the drug/receptor complex to bind specific response elements in the promoter regions of specific genes. In ophthalmic applications, chronic treatment of corticosteroids has been associated with side effects such as increased intraocular pressure (IOP) and increased risk of cataracts development (McGhee et al., 2002). Consequently, an unmet medical need exists for novel steroids formulations that promote remarkable anti-inflammatory activity, minimizing side effects. The use of topical "soft" steroids, such as loteprednol, has a lower likelihood of increasing IOP and inducing cataract formation (McGhee et al., 2002; Sheppard et al., 2016), even though these complications cannot be ruled out. Cortisol (called hydrocortisone, when used as a drug) is the main glucocorticoid with low anti-inflammatory action and short duration of action. Based on these premises, the purpose of the present study was to investigate the ocular pharmacokinetic/pharmacodynamics (PK/ $\mathrm{PD})$ profile of $\mathrm{HC}$ using in vitro and in vivo models of dry eye.

\section{METHODS}

\section{In Vitro Studies}

\section{Hyperosmotic Stress}

Confluent rabbit corneal epithelial cells (SIRCs) at 16 days were exposed to an equal volume ( $0.5 \mathrm{ml} /$ well $)$ of serum-free medium (SHEM without FBS) for $24 \mathrm{~h}$ and then treated for $24 \mathrm{~h}$ with hyperosmolar media $(450 \mathrm{mOsm} / \mathrm{L})$ with or without $0.001 \%$ hydrocortisone treatment. The osmolarity value was chosen based on previous studies indicating a threshold of $450 \mathrm{mOsm} / \mathrm{l}$ for the induction of cell damage (Liu et al., 2009). The osmolarity of the culture media was assessed by an osmometer (Osmomat 30 Gonotech, Berlin, Germany). After 24 h, the levels of TNF $\alpha$, TNF-related apoptosis-inducing ligand (TRAIL), IL-1 $\beta$, and IL-8 were determined with ELISA kits (R\&D System, Milan, Italy; RayBiotech, Milan, Italy) following the manufactures' protocols. We also carried out a western blot assay to measure TRAIL expression in the cell lysates. Cells were harvested in RIPA lysis buffer supplemented with protease and phosphatase inhibitors cocktail (Sigma-Aldrich). After centrifugation at $14,000 \mathrm{rpm}$ at $4^{\circ} \mathrm{C}$ for $10 \mathrm{~min}$, the supernatants were collected. The total protein concentration in the supernatant was determined using the Bradford reagent (Bio-Rad Laboratories, Segrate, Italy) and measuring absorbance with a VarioskanTM Flash Multimode Reader. Equal amounts of protein $(30 \mu \mathrm{g})$ were resolved by $8-12 \%$ (10\%) SDS-PAGE and then transferred to Hybond ECL nitrocellulose membranes (GE Healthcare, Little Chalfont, UK). Membranes were blocked with 5\% nonfat dry milk in phosphate-buffered saline plus $0.1 \%$ Tween 20 (PBST) (Bio-Rad Laboratories, Segrate, Italy) and then incubated overnight at $4^{\circ} \mathrm{C}$ with rabbit anti-TRAIL/TNFSF10 polyclonal antibody (Abcam, cat. No. ab2435; 1:200). The membranes were then washed with PBS-T and finally probed with horseradish peroxidase-conjugated antirabbit IgG secondary antibody (GE Healthcare, cat. No. GENA934; 1:5000) for $1 \mathrm{~h}$ at RT in 5\% nonfat dry milk. Detection of specific bands was carried out using the iBright Imaging Systems (Thermo Fisher Scientific, Inc.) after 
enhanced chemiluminescence (ECL) (GE Healthcare). $\beta$-actin (Santa Cruz Biotechnology, sc-47778; 1:1000) was used as the housekeeping protein. Densitometric analysis of band intensity was carried out by the ImageJ software (https://imagej.nih.gov/ ij/). All experiments were repeated at least four times, each run in triplicate.

\section{Scratch-Wound Assay}

A scratch-wound assay on SIRCs was used to assess the effects of $\mathrm{HC}$ on wound areas. SIRCs were purchased from ATCC ${ }^{\circledR}$ and cultured in Eagle's minimum essential medium (EMEM, SigmaAldrich) with $10 \%$ of FBS (fetal bovine serum), 1X MEM NEAA (minimum essential medium nonessential amino acids) and $1 \mathrm{X}$ $\mathrm{P} / \mathrm{S}$ (penicillin/streptomycin). Cellular adhesion was promoted applying 5-10 $\mu \mathrm{l}$ gelatin solution $/ \mathrm{cm}^{2}$ (i.e., $0.1-0.2 \mathrm{mg} / \mathrm{cm}^{2}$ gelatin) as plate coating agent. Cells were incubated at $37^{\circ} \mathrm{C}$ in $5 \%$ $\mathrm{CO} 2$ in humidified air. Cells were seeded $(1.7 \times 105)$ on 24 -well culture plates (Corning ${ }^{\circledR}$ ) and cultured until confluence, as previously described. After confluence, cells were washed twice with warm phosphate saline buffer (PBS, 1X) and then incubated with serum-free medium for $5 \mathrm{~h}$ (starvation). Then, the SIRC monolayer was scratched with a sterile $200 \mu \mathrm{l}$ pipette tip, and the wells were washed with fresh medium to remove detached cells before incubation in serum-free medium containing sodium hyaluronate $0.2 \%$ and $\mathrm{HC}(0.001 \%)$. Pictures of the wound areas were taken and the coordinates noted at starting time of experiment (T0) and forty-eight hours (T48) after scratch. T0 and T48 wound images were obtained with an inverted optical microscope (IM3 Optika), 20X magnification. Four images per condition were analyzed and mean wound area was measured with the image analysis system Image J Software (https://imagej. nih.gov/ij/). We reported the percentage of wound closure, as normalized to the corresponding wound area at T0.

\section{In Vivo Studies}

\section{Animals}

Male New Zealand albino rabbits $(1.8-2.0 \mathrm{~kg})$ were purchased from Envigo (Udine, Italy). Animals were housed under standard conditions with food and water provided ad libitum in a lightcontrolled room and set temperature and humidity. Animal care and experimental procedures were carried out according to the ARVO Statement for the Use of Animals in Ophthalmic and Vision Research. Protocols were approved by the Institutional Animal Care and Use Committee of the University of Catania (project \#303).

\section{Ocular Distribution}

Four eye drops containing hydrocortisone $(\mathrm{FA}=0.001 \%, \mathrm{FB}=$ $0.003 \%, \mathrm{FC}=0.005 \%$, and $\mathrm{FD}=0.33 \%)$ and sodium hyaluronate $(0.2 \%)$ were administered in the conjunctival sac four times every $2 \mathrm{~h}$ into the rabbit eye. Aqueous humor samples $(\mathrm{n}=$ 4 for each time point) were collected after 15, 30, 60, and 90 min from the last instillation and hydrocortisone detected by liquid chromatography tandem-mass spectrometry (LCMS/MS). The internal standard prednisolone (5 $\mu \mathrm{l}$ of $2 \mu \mathrm{g} /$ $\mathrm{ml}$ solution) was added to $100 \mu \mathrm{l}$ of aqueous humor samples. Ethyl acetate was then added, and the samples were vortexed, centrifuged $\left(12,000 \mathrm{rpm} 25 \mathrm{~min} 4^{\circ} \mathrm{C}\right)$, and dried. After drying, 100- $\mu$ l HPLC mobile phase was added to samples, subjected to vortexing and spinning. Samples were then injected into LC-MS/MS (Agilent 6410A Triple Quadrupole). We determined the limit of detection method, which was $2.5 \mathrm{ng} /$ $\mathrm{ml}$ for $\mathrm{HC}$. The data generated by the present study were used to choose the right HC concentration to be studied for the evaluations of pharmacodynamics.

\section{Dry Eye Models}

Two different paradigms were used to investigate the ocular pharmacodynamics profile of hydrocortisone: aqueous tear deficiency model and lacrimal gland inflammation-induced dry eye model elicited by atropine and concanavalin A (ConA).

Atropine sulfate (1.0\%) eye drops (Atropina Farmigea, Farmigea, Pisa, Italy) was instilled into the lower conjunctival sac of the eye (four times within $12 \mathrm{~h}$ ). Atropine is a muscarinic $\left(\mathrm{M}_{3}\right)$ receptor antagonist, which is able to decrease tear volume and alter tear film stability. Fifteen minutes after atropine administration, we treated the eyes with $0.001 \% \mathrm{HC}$ eye drops or vehicle (four times within $12 \mathrm{~h}$ ). To assess the degree of dry eye after $24 \mathrm{~h}$ from the atropine instillation, tear volume and tear film breakup time (TBUT) were used as endpoints. Tear volume was evaluated by Schirmer strips (Eagle Vision, Memphis, TN, USA). The strips were carefully placed in the posterior (i.e., temporal) lower fornix for $60 \mathrm{~s}$, and the wetted area was evaluated. TBUT was assessed after instillation $(5 \mu \mathrm{l})$ of sodium fluorescein (2\%) in the rabbit eye. A slit lamp (Sbisà, Firenze, Italy) with cobalt blue filter was used to enhance fluorescein patterns; TBUT was determined by measuring the time from the opening of the eyes until the appearance of the first black spot or streak on the cornea. The procedure was sequentially performed three times and the average of three readings per eye was calculated. Data were analyzed by two investigators who were unaware of the experimental design. Two groups of animals ( $n=6$ /group) were used and treated as follows: (1) control group (atropine treatment + eye drops vehicle) and treated group (atropine treatment $+0.001 \% \mathrm{HC}$ ).

A separate set of rabbits was treated with the T-cell mitogen ConA $(300 \mu \mathrm{g} / 50 \mu \mathrm{l}$ of BSS) in the lacrimal glands using a 28-gauge needle. Before ConA injection, the animals were anesthetized by dexmedetomidine $(20 \mu \mathrm{g} / \mathrm{kg}$ intramuscularly; Dexdomitor ${ }^{\mathrm{TM}}$,Vetoquinol, Bertinoro, Italy) and tiletamine + zolazepam (10 mg/kg intramuscularly; Zoletin ${ }^{\mathrm{TM}}$, Virbec, Milan Italy). Control rabbits were injected with $50 \mu \mathrm{l}$ of BSS in the lacrimal gland. Rabbits were randomized into two treatment groups ( $\mathrm{n}=5$ per group) and instilled with ophthalmic solution four times a day for 3 days with either drug $(0.001 \% \mathrm{HC})$ or vehicle. After 3 days from ConA injection, tears were collected and TNF- $\alpha$, IL-8, and MMP-9 were measured following the manufactures' protocols (R\&D System, Milan, Italy; RayBiotech, Milan, Italy). A separate set of animals was used to assess the safety profile, in terms of intraocular pressure, of the ophthalmic formulation with the lowest concentration of the drug $(0.001 \%$ $\mathrm{HC}$ ). One drop was instilled into the conjunctival sac (four times within $12 \mathrm{~h}$ for 4 weeks), and IOP measured every week by TonoPen (Reichert, Buffalo, NY, USA). 


\section{Statistical Analysis}

The data were expressed as mean \pm SD. Statistical analysis was conducted using ANOVA followed by Dunnett's test. Student's t-test was performed where appropriate. A $p$-value $<0.05$ was predetermined as the criterion of statistical significance.

\section{RESULTS}

\section{In Vitro Studies}

Tear film osmolarity is an important causative factor in the pathogenesis of DED. Tear hyperosmolority resulting from decreased lacrimal flow contributes to ocular surface damage through a cascade of inflammatory events. For this reason, we used an in vitro paradigm that mimics this clinical condition. We induced corneal cell damage by a hyperosmotic environment and assessed the effects of a low concentration (0.001\%) of HC on cytokine expression. The corneal cells exposed to hyperosmotic insult for $24 \mathrm{~h}$ showed a significant $(\mathrm{p}<0.05)$ increase of TNF $\alpha$, IL-1 $\beta$, and IL- 8 levels that were significantly $(\mathrm{p}<0.05)$ counteracted by HC treatment (Figure 1).

We assessed TRAIL expression in SIRCs exposed to a hyperosmolarity condition w/o $0.001 \%$ HC (Figure 2). Hyperosmotic insult increased significantly $(\mathrm{p}<0.05)$ the expression of TRAIL in SIRCs, compared to control cells (300 mOsm). Furthermore, hydrocortisone treatment significantly $(\mathrm{p}<0.05)$ increased TRAIL expression compared to SIRCs exposed to hyperosmotic stress (Figure 3A). In order to assess if TRAIL levels in the SIRC medium were in the protective range $(\leq 10 \mathrm{ng} / \mathrm{ml})$, we measured soluble TRAIL by ELISA. Medium TRAIL content in SIRCs (control, $300 \mathrm{mOsm}$ ) was very low and increased with either a hyperosmotic insult $(27 \mathrm{pg} / \mathrm{ml})$ or $\mathrm{HC}$ treatment $(57 \mathrm{pg} / \mathrm{ml})$ that means five logs below the toxic cutoff (100 ng/ml; Lee et al., 2002) (Figure 2B).

Furthermore, the wound healing assay was performed on the corneal cell monolayer. Migration ability was assessed in a scratch assay on the SIRC monolayer. Microscopic bright field pictures were taken, and wound areas were evaluated at $48 \mathrm{~h}$ and normalized to areas at T0. HC at $0.001 \%$ displayed a statistically significant $(\mathrm{p}<0.05)$ effect on wound healing compared to controls (Figure 3). Indeed, $\mathrm{HC}$ at the tested concentration did not influence the wound healing of damaged corneal epithelial cells.

\section{In Vivo Studies}

\section{Ocular Distribution}

This study was important in order to choose the right dose to carry out the pharmacodynamics study. We determined the PK profile of four $\mathrm{HC}$ eye drops, tested at four different concentrations $(\mathrm{FA}=0.001 \%, \mathrm{FB}=0.003 \%, \mathrm{FD}=0.005 \%$, and $\mathrm{FC}=0.33 \%)$. No levels, at any time, of hydrocortisone were detected in the aqueous humor in the group treated with the formulation (FA) containing the lowest drug concentration (0.001\%). On the contrary, the aqueous humor samples obtained from the other three groups $(0.003 \%, 0.005 \%$, and $0.33 \%)$ showed remarkable levels of hydrocortisone (Table 1, Figure 4). We demonstrated

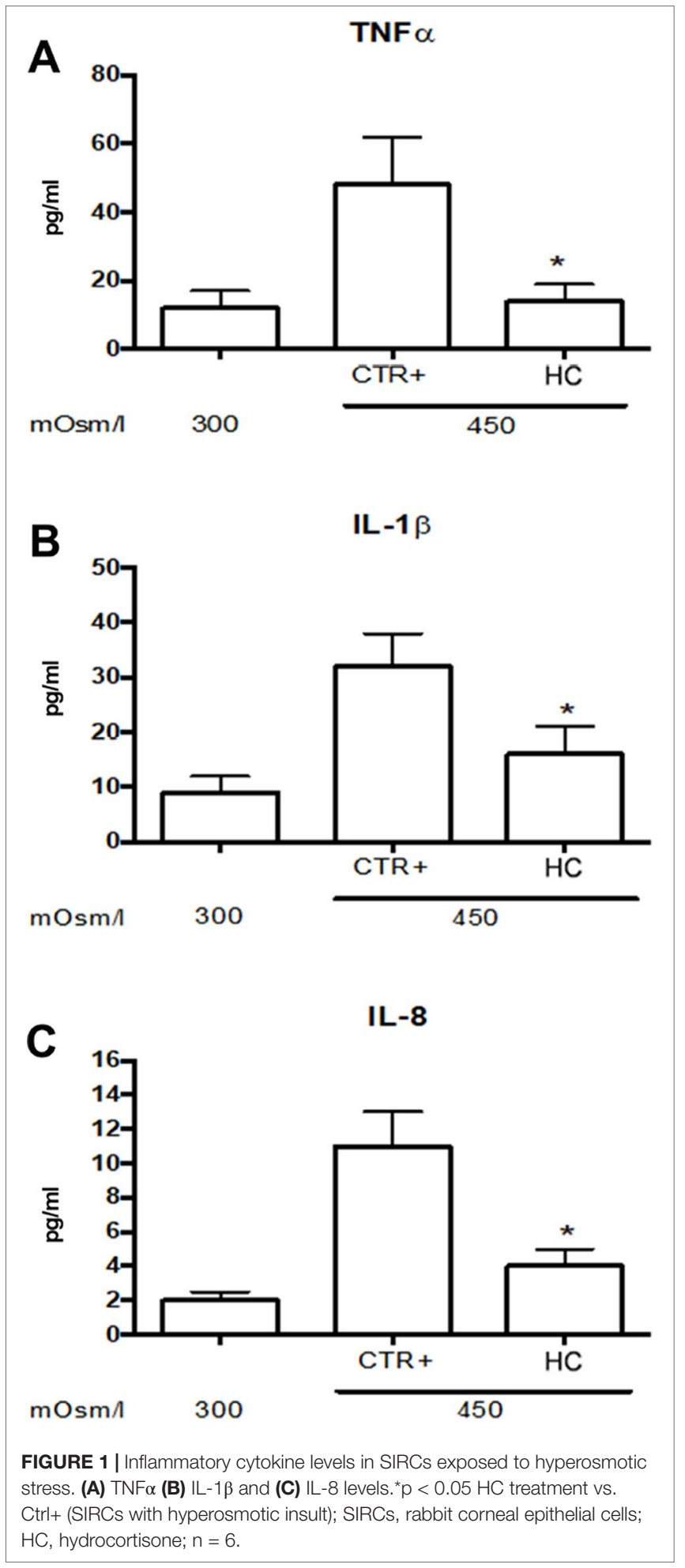

that $\mathrm{HC}$ crosses the corneal barrier, in a dose-dependent manner (see the $\mathrm{C}_{\max }$ and $\mathrm{AUC}_{0-90}$ values, Table 1) Our data confirmed that $\mathrm{HC}$ at the lowest dose $(0.001 \%)$ does not cross the corneal barrier; therefore, the risk of side effects, such as intraocular pressure rise, is negligible. This allowed us to choose the right $\mathrm{HC}$ concentration to be studied for the evaluations of efficacy. 


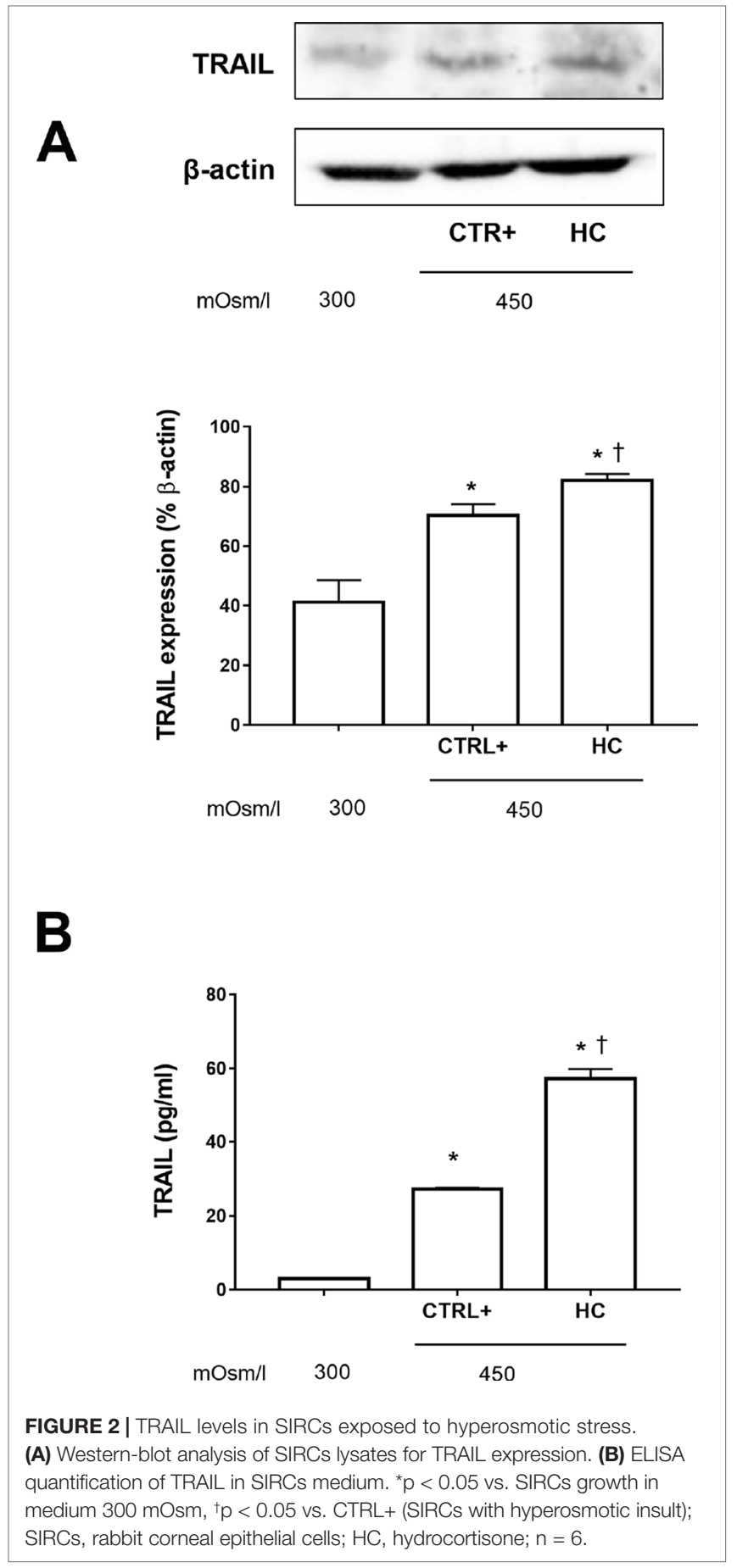

\section{In Vivo Efficacy Study}

In the atropine DED animal model, we assessed tear volume and tear film quality after $0.001 \% \mathrm{HC}$ treatment, with Schirmer's test and TBUT, which are currently used in clinical practice to diagnose dry eye and assess severity grade (Barabino et al., 2004).

Tear volume and tear film integrity were measured, before $(13.5 \pm 0.5 \mathrm{~mm}$ and $>60 \mathrm{~s}$, respectively) and after $24 \mathrm{~h}$ from the first atropine instillation, by Schirmer's test and TBUT, respectively. The baseline values of tear volume and TBUT

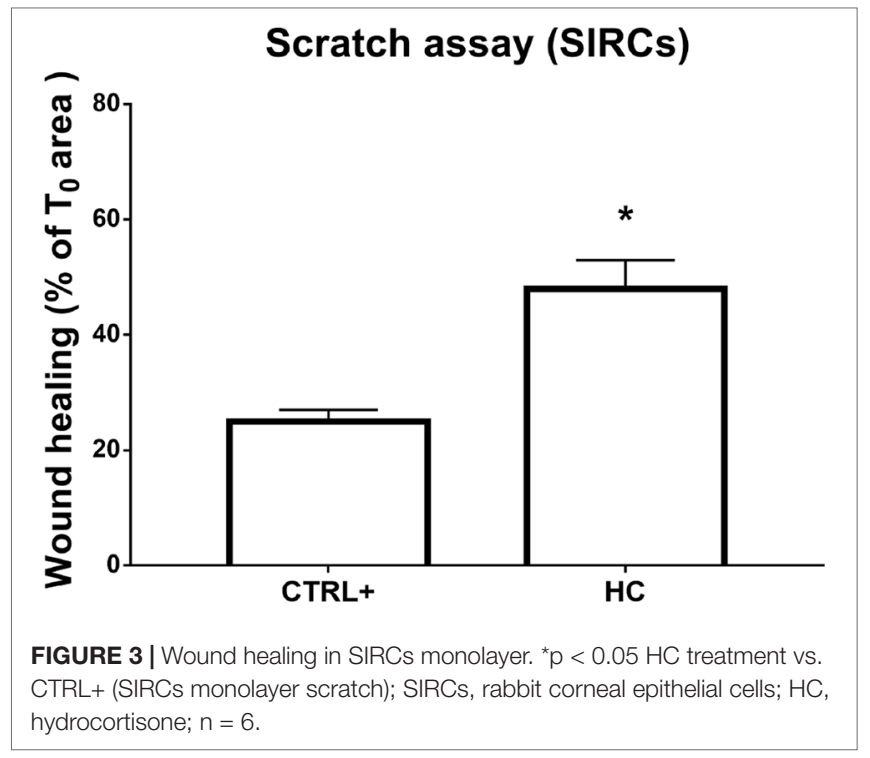

were in accordance with previous study (Shafiee et al., 2011). Multiple topical administrations of atropine reduced tear volume (around $5 \mathrm{~mm}$ ) in the vehicle-treated group; this effect was significantly $(\mathrm{p}<0.01)$ counteracted by topical treatment with hydrocortisone $(0.001 \%)$ eye drops (Figure 5A). A similar trend was also observed in terms of tear film integrity as measured by TBUT. Vehicle-treated eyes showed a marked and immediate reduction in TBUT that started at day 1 (reduction of TBUT to around $10 \mathrm{~s}$ ) and continued at day 3 (TBUT around $8 \mathrm{~s}$ ) (Figure 5B). In contrast, HC eye drops significantly ( $\mathrm{p}<$ 0.01 ) preserved the tear film integrity at $24 \mathrm{~h}$ from atropine instillation (Figure 5B). Finally, repeated topical administration of the ophthalmic formulation at the lowest dose of $\mathrm{HC}$ showed no change in terms of IOP values throughout the 4 weeks (data not shown).

A lacrimal gland inflammation-induced dry eye model was used in a separate set of animals. Rabbits received a ConA (T-cell mitogen) injection into the lacrimal gland. Tear volume and tear film quality were affected in the ConA animal model of DED and $0.001 \% \mathrm{HC}$ treatment reverted tear volume and TBUT to control levels (CTRL+ = injected with ConA; no treatment with HC) (Figure 6A). After 3 days, inflammatory biomarkers were measured in the tear samples. Topical treatment with hydrocortisone $(0.001 \%)$ significantly $(\mathrm{p}<0.05)$ reduced the tear levels of TNF- $\alpha$, IL-8, and MMP-9 compared with the vehicle group. Topical treatment with hydrocortisone $(0.001 \%)$ significantly $(\mathrm{p}<0.05)$ counteracted the tear volume reduction and tear integrity at all the time points (Figure 6B).

\section{DISCUSSION}

The production of tears is regulated to maintain tear osmolarity within narrow limits at all times (Sullivan et al., 2012). Tear film osmolarity dysregulation is one of the key factors in the pathogenesis of DED, including Sjögren syndrome, a chronic autoimmune disorder. Tear hyperosmolarity, resulting from 
TABLE 1 | Aqueous distribution of hydrocortisone after instillation of four formulations in the rabbit eye: PK parameters.

\begin{tabular}{|c|c|c|c|}
\hline$\%$ w/v hydrocortisone & $\mathbf{C}_{\max }(\mathbf{n g} / \mathbf{m l})$ & $\mathbf{T}_{\max }(\min )$ & AUC $_{0-90^{\prime}},(\mathbf{n g} \cdot \mathrm{min} / \mathrm{ml})$ \\
\hline $0.001 \%$ & - & - & - \\
\hline $0.003 \%$ & $10.6 \pm 2.8$ & 30 & $535.1 \pm 65.9^{\star}$ \\
\hline $0.005 \%$ & $48.94 \pm 1.14$ & 60 & $2417 \pm 50^{\star}$ \\
\hline $0.33 \%$ & $69.15 \pm 1.00$ & 30 & $4510 \pm 30^{\star}$ \\
\hline
\end{tabular}

${ }^{*} p<0.05$ for TWO-WAY ANOVA comparison between treatment groups.

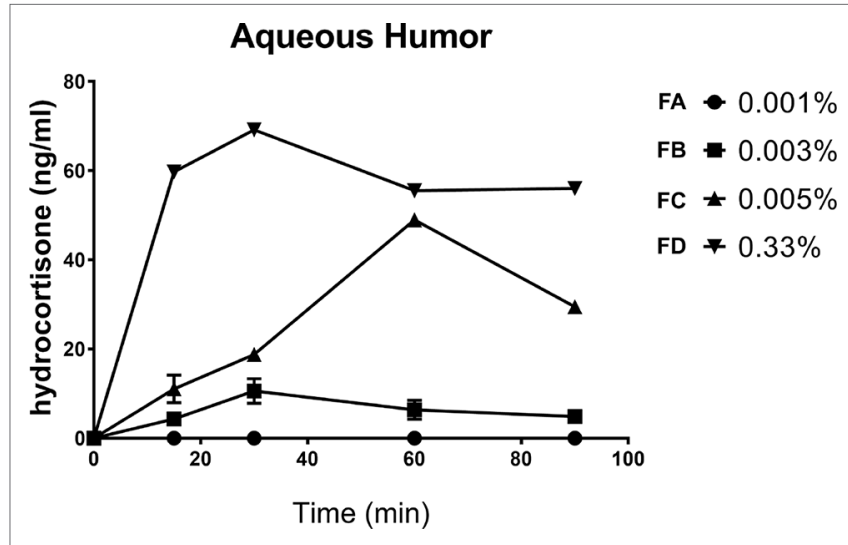

FIGURE 4 | Aqueous distribution of hydrocortisone after instillation of four formulations in the rabbit eye.

decreased lacrimal flow or tear film instability, contributes to ocular surface damage both directly and, indirectly, through a cascade of inflammatory events. Physiological levels of tear film osmolarity are in the range of $302 \pm 9.7 \mathrm{mOsm} / \mathrm{l}$ (Tomlinson et al., 2006). Meanwhile, the highest values for tear osmolarity measured in DED patients are below $500 \mathrm{mOsm} / \mathrm{l}$. However, it is likely that the levels achieved at the ocular surface are much higher than $500 \mathrm{mOsm} / \mathrm{l}$, particularly at the cornea sites where tear film starts to breakup.

Low aqueous tear flow and/or excessive evaporation induce hyperosmolarity, eliciting the release of inflammatory mediators into the tear fluid. Clinical studies consistently report upregulation of several pro-inflammatory mediators in the tears of DED patients such as interleukins, cytokines, and metalloproteinases (Lin et al., 2010; Zhang et al., 2011). The last group is composed by endopeptidases involved in tissue remodeling, and elevated levels of MMP-9 have been shown to be diagnostic in clinical evaluations of DED. It is well known that desiccating and hyperosmolarity stresses induce expression of $\mathrm{NF} \kappa \mathrm{B}$, which is involved in signaling and subsequent inflammatory alterations related to DED. The NFkB signaling pathway regulates the expression of cytokines and MMPs, such as MMP-9, which is involved in the pathogenesis of DED.

In general, inflammation is an adaptive response of the immune system to noxious insults to maintain homeostasis and restore functionality. Recently, a new concept of the origin of inflammation suggested that, between basal homeostatic conditions and canonical inflammation, there is a condition called “para-inflammation”(Medzhitov, 2008). Para-inflammation

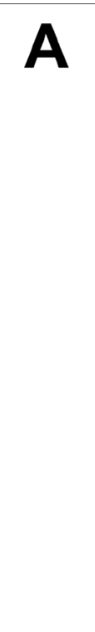

B

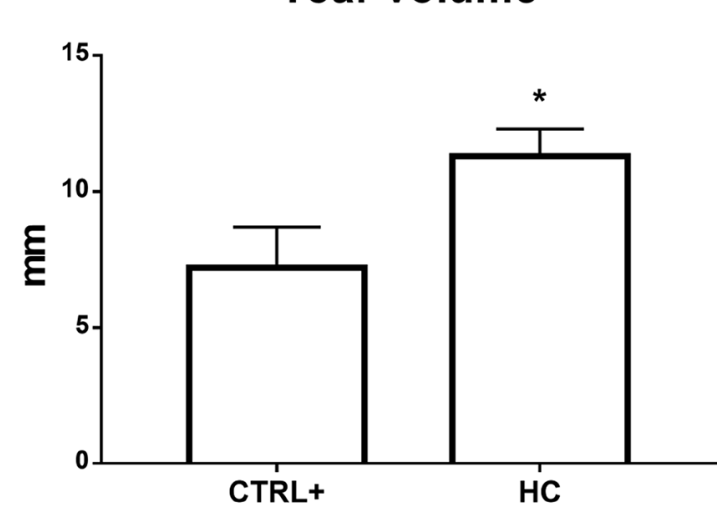

TBUT

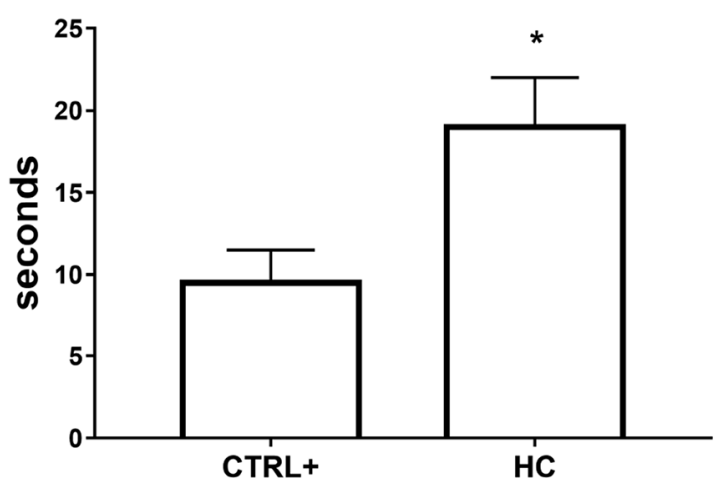

FIGURE 5 | Tear volume (A) and tear film stability (B) in atropine induced dry eye model. TBUT, tear breakup time. ${ }^{*} \mathrm{p}<0.05 \mathrm{HC}$ vs. CTRL+ (eye treated with atropine; no treatment with $\mathrm{HC}$ ); $\mathrm{HC}$, hydrocortisone; $\mathrm{n}=6$.

is an adaptive response of the immune system to low levels of tissue damage (i.e., a low degree of "danger" stimuli), such as in DED where hyperosmotic stress accumulates bit by bit for years. The physiological role of para-inflammation is to maintain homeostasis (or reset the homeostatic threshold of the tissue) and restore tissue functionality, even though parainflammatory processes could be dysregulated, contributing to corneal damage. Therefore, the new concept is that a wellcontrolled para-inflammation is beneficial, while a dysregulated para-inflammation is detrimental. In this context, the protein TRAIL plays an important role in the modulation of autoimmune inflammation. TRAIL is a protein known for its 

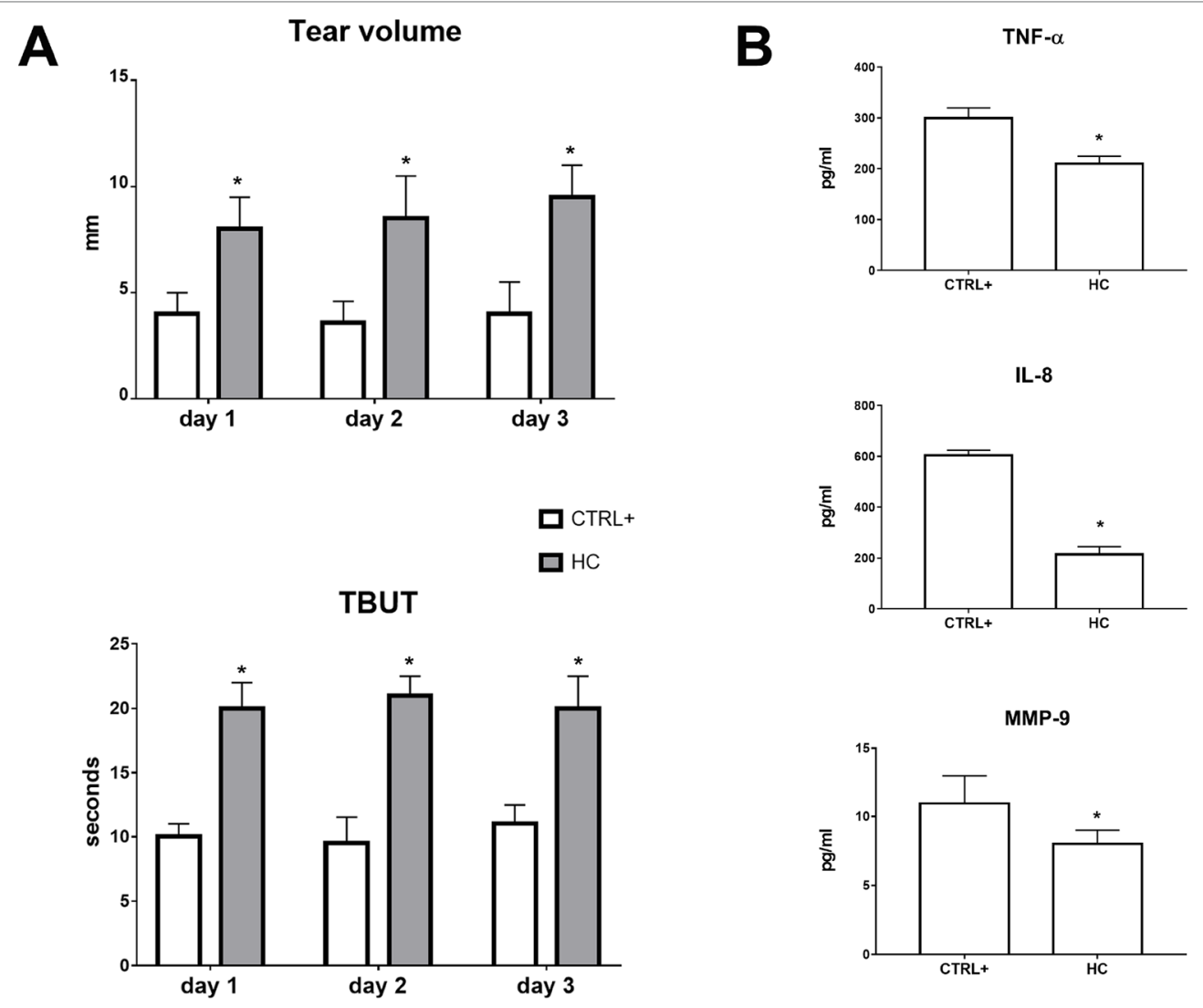

FIGURE 6 | Tear volume, tear film stability (A), and inflammatory biomarkers (B) in ConA induced dry eye model. TBUT, tear breakup time. ${ }^{*} \mathrm{p}<0.05 \mathrm{HC}$ vs. CTRL+ (eye treated with atropine; no treatment with $\mathrm{HC}$ ); $\mathrm{HC}$, hydrocortisone; ConA, concanavalin $\mathrm{A} ; \mathrm{n}=6$.

ability to promote cell death and is constitutively expressed in ocular tissues including the cornea (Lee et al., 2002; Narayanan et al., 2006). However, several groups have recently shown that TRAIL can also stimulate cell proliferation (Kavurma et al., 2008; Chan et al., 2010), with anti-inflammatory effects (Manuneedhi Cholan et al., 2018). Indeed, these reports suggest a protective role of TRAIL, even though the precise mechanism/s are not clear yet. This dual effect could be related to several factors such as, among others, different milieu, diverse insults, and various grades of damage.

In the present study, we observed an increase of inflammatory biomarkers such as TNF- $\alpha$, IL- $1 \beta$ and IL- 8 in corneal cells after hyperosmotic stress that were attenuated by $\mathrm{HC}$ treatment at a very low concentration. Furthermore, we found that TRAIL expression was increased in corneal cells exposed to hyperosmotic stress also in the presence of very low concentrations of $\mathrm{HC}$; interestingly, the TRAIL levels detected were in a concentration range known to be protective ( $\leq 10 \mathrm{ng} / \mathrm{ml}$ ) (Hilliard et al., 2001; Cantarella et al., 2014; Di Bartolo et al., 2015; Cartland et al., 2016). These findings support the hypothesis that a corneal physiological mechanism of defense is triggered when the cells are undergoing osmotic insult and that this protective pathway is facilitated by HC. It is noteworthy that TRAIL controls autoimmune inflammation, and Hilliard et al. (2001) demonstrated that TRAIL inhibits experimental autoimmune encephalomyelitis by preventing activation of autoimmune $\mathrm{T}$ cells (Hilliard et al., 2001). This point is important considering that $\mathrm{T}$ cells play a major role in the detrimental inflammatory process that occurs in the lacrimal glands of Sjögren syndrome patients (Moutsopoulos, 2014).

In summary, our in vivo data demonstrate that $0.001 \%$ HC eye drops are an effective anti-inflammatory therapy when tested in an aqueous tear deficiency model (atropineinduced dry eye) and lacrimal gland inflammation-induced dry eye model (ConA-induced dry eye) in rabbits. The present study demonstrated, both in vitro and in vivo, that the antiinflammatory action of $\mathrm{HC}$ on the ocular surface is related to the modulation of TNF $\alpha$, TRAIL, IL-1 $\beta$, IL-8, and MMP-9. The quenching action of $\mathrm{HC}$ on the inflammatory process brought about a restoration of ocular tissues, tear film as demonstrated, among others, by Schirmer's test and TBUT. Finally, the pharmacokinetics study demonstrated that after topical administration of the ophthalmic formulation, containing the lowest dose of HC $(0.001 \%)$, in the rabbit eye, no trace of the drug was detected in the aqueous humor. Altogether, these data suggest that hydrocortisone, at a very low concentration, 
has a relevant anti-inflammatory effect on both in vitro and in vivo dry eye models, with a good impact in terms of safety, warranting further clinical evaluation.

\section{DATA AVAILABILITY STATEMENT}

All datasets generated for this study are included in the manuscript/supplementary files.

\section{ETHICS STATEMENT}

The animal study was reviewed and approved by University of Catania.

\section{REFERENCES}

Aptel, F., Colin, C., Kaderli, S., Deloche, C., Bron, A. M., Stewart, M. W., et al. (2017). Management of postoperative inflammation after cataract and complex ocular surgeries: a systematic review and Delphi survey. Br. J. Ophthalmol. 101 (11), 1-10. doi: 10.1136/bjophthalmol-2017-310324

Barabino, S., Chen, W., and Dana, M. R. (2004). Tear film and ocular surface tests in animal models of dry eye: Uses and limitations. Exp. Eye Res. 79 (5), 613-621. doi: 10.1016/j.exer.2004.07.002

Bekhbat, M., Rowson, S. A., and Neigh, G. N. (2017). Checks and balances: The glucocorticoid receptor and NFkB in good times and bad. Front. Neuroendocrinol. 46, 15-31 doi: 10.1016/j.yfrne.2017.05.001

Bron, A. J., de Paiva, C. S., Chauhan, S. K., Bonini, S., Gabison, E. E., Jain, S., et al. (2017). TFOS DEWS II pathophysiology report. Ocul. Surf. 15 (3), 438-510. doi: $10.1016 /$ j.jtos.2017.05.011

Bucolo, C., Musumeci, M., Musumeci, S., and Drago, F. (2011). Acidic mammalian chitinase and the eye: Implications for ocular inflammatory diseases. Front. Pharmacol. (7), 2-43 doi: 10.3389/fphar.2011.00043

Caceres-del-Carpio, J., Costa, R. D., Haider, A., Narayanan, R., and Kuppermann, B. D. (2016). Corticosteroids: Triamcinolone, Dexamethasone and Fluocinolone. Dev. Ophthalmol. 55, 221-231. doi: 10.1159/000431198

Cantarella, G., Pignataro, G., Di Benedetto, G., Anzilotti, S., Vinciguerra, A., Cuomo, O., et al. (2014). Ischemic tolerance modulates TRAIL expression and its receptors and generates a neuroprotected phenotype. Cell Death Dis. 5, e1331 doi: 10.1038/cddis.2014.286

Cartland, S. P., Genner, S. W., Zahoor, A., and Kavurma, M. M. (2016). Comparative evaluation of trail, FGF-2 and VEGF-A-Induced angiogenesis in vitro and in vivo. Int. J. Mol. Sci. 17 (12), pii:E2025. doi: 10.3390/ijms17122025

Chan, J., Prado-Lourenco, L., Khachigian, L. M., Bennett, M. R., Di Bartolo, B. A., and Kavurma, M. M. (2010). TRAIL promotes VSMC proliferation and neointima formation in a FGF-2-, Sp1 Phosphorylation-, and NFkB-Dependent Manner. Cir. Res. 106 (6), 1061-71 doi: 10.1161/ CIRCRESAHA.109.206029

Craig, J. P., Nichols, K. K., Akpek, E. K., Caffery, B., Dua, H. S., Joo, C. K., et al. (2017). TFOS DEWS II Definition and Classification Report. Ocul. Surf. doi: 10.1016/j.jtos.2017.05.008

Di Bartolo, B. A., Cartland, S. P., Prado-Lourenco, L., Griffith, T. S., Gentile, C., Ravindran, J., et al. (2015). Tumor necrosis factor-related apoptosisinducing ligand (TRAIL) promotes angiogenesis and ischemia-induced neovascularization via NADPH oxidase 4 (NOX4) and nitric oxidedependent mechanisms. J.Am. Heart Assoc. 4 (11), pii: e002527. doi: 10.1161/ JAHA.115.002527

Ervin, A. M., Law, A., and Pucker, A. D. (2017). Punctal occlusion for dry eye syndrome. Cochrane Database Syst. Rev. 6, CD006775 doi: 10.1002/14651858. CD006775.pub3

Hilliard, B., Wilmen, A., Seidel, C., Liu, T.-S. T., Göke, R., and Chen, Y. (2001). Roles of TNF-Related Apoptosis-Inducing Ligand in Experimental

\section{AUTHOR CONTRIBUTIONS}

Authors that made substantial contributions to conception and design: $\mathrm{CB}, \mathrm{SB}$, and FD. Authors that made contribution to acquisition of data: CB, AF, CGF, FL, CBMP, GC, GB, CBurg, and $\mathrm{CP}$. Authors that made contribution to statistical analysis and interpretation of data: CB, CBMP, GC, and RB. Authors that participated in drafting the article and revising it critically: $\mathrm{CB}$, $\mathrm{GC}, \mathrm{RB}, \mathrm{SB}$, and FD.

\section{ACKNOWLEDGMENTS}

"Piano Triennale Per La Ricerca-Linea Intervento 2, University of Catania, Italy”.

Autoimmune Encephalomyelitis. J. Immunol. 166 (2), 1314-1319. doi: 10.4049/ jimmunol.166.2.1314

Kavurma, M. M., Schoppet, M., Bobryshev, Y. V., Khachigian, L. M., and Bennett, M. R. (2008). TRAIL stimulates proliferation of vascular smooth muscle cells via activation of NF- $\mathrm{BB}$ and induction of insulin-like growth factor-1 receptor. J. Biol. Chem. 283(12), 7754-62 doi: 10.1074/jbc.M706927200

Lee, H., Herndon, J. M., Barreiro, R., Griffith, T. S., and Ferguson, T. A. (2002). TRAIL: A Mechanism of Tumor Surveillance in an Immune Privileged Site. J. Immunol. 169 (9), 4739-4744. doi: 10.4049/jimmunol.169.9.4739

Lessard, C. J., Li, H., Adrianto, I., Ice, J. A., Rasmussen, A., Grundahl, K. M., et al. (2013). Variants at multiple loci implicated in both innate and adaptive immune responses are associated with Sjögren's syndrome. Nat. Genet. 45 (11), 1284-1292. doi: 10.1038/ng.2792

Lin, H., Li, W., Dong, N., Chen, W., Liu, J., Chen, L., et al. (2010). Changes in corneal epithelial layer inflammatory cells in aqueous tear-deficient dry eye. Invest. Ophthalmol. Vis. Sci. 51 (1), 122-128. doi: 10.1167/iovs.09-3629

Liu, H., Begley, C., Chen, M., Bradley, A., Bonanno, J., McNamara, N. A., et al. (2009). A link between tear instability and hyperosmolarity in dry eye. Invest. Ophthalmol. Vis. Sci. 50 (8), 3671-3679. doi: 10.1167/iovs.08-2689

Liu, Z., and Pflugfelder, S. C. (1999). Corneal surface regularity and the effect of artificial tears in aqueous tear deficiency. Ophthalmology. 106 (5), 939-943. doi: 10.1016/S0161-6420(99)00513-8

Manuneedhi Cholan, P., Cartland, S. P., Dang, L., Rayner, B. S., Patel, S., Thomas, S. R., et al. (2018). TRAIL protects against endothelial dysfunction in vivo and inhibits angiotensin-II-induced oxidative stress in vascular endothelial cells in vitro. Free Radic. Biol. Med. 126, 341-349. doi: 10.1016/j.freeradbiomed.2018.08.031

McGhee, C. N. J., Dean, S., and Danesh-Meyer, H. (2002). Locally administered ocular corticosteroids benefits and risks. Drug Safety. 25 (1), 33-55. doi: 10.2165/00002018-200225010-00004

Medzhitov, R. (2008). Origin and physiological roles of inflammation. Nature. 454 (7203), 428-435. doi: 10.1038/nature07201

Moutsopoulos, H. M. (2014). Sjögren's syndrome: A forty-year scientific journey. J. Autoimmunity. 51, 1-9 doi: 10.1016/j.jaut.2014.01.001

Musumeci, M., Bellin, M., Maltese, A., Aragona, P., Bucolo, C., and Musumeci, S. (2008). Chitinase levels in the tears of subjects with ocular allergies. Cornea. 27 (2), 168-173. doi: 10.1097/ICO.0b013e318159a950

Narayanan, S., Miller, W. L., and McDermott, A. M. (2006). Conjunctival cytokine expression in symptomatic moderate dry eye subjects. Invest. Ophthalmol. Vis. Sci. doi: 10.1167/iovs.05-1364

Pinto-Fraga, J., Lopez-Miguel, A., Gonzalez-Garcia, M. J., Fernandez, I., Lopez-De-La-Rosa, A., Enriquez-De-Salamanca, A., et al. (2016). Topical Fluorometholone Protects the Ocular Surface of Dry Eye Patients from Desiccating Stress: A Randomized Controlled Clinical Trial. Ophthalmology. 124 (2), e14. doi: 10.1016/j.ophtha.2015.09.029

Pucker, A. D., Ng, S. M., and Nichols, J. J. (2016). Over the counter (OTC) artificial tear drops for dry eye syndrome. Cochrane Database Syst. Rev. 2, CD009729 doi: 10.1002/14651858.CD009729.pub2 
Ridolo, E., Montagni, M., Caminati, M., Senna, G., Incorvaia, C., and Canonica, G. W. (2014). Emerging drugs for allergic conjunctivitis. Expert Opin. Emerg. Drugs. 19 (2), 291-302. doi: 10.1517/14728214.2014.902443

Shafiee, A., Bucolo, C., Budzynski, E., Ward, K. W., and López, F. J. (2011). In vivo ocular efficacy profile of mapracorat, a novel selective glucocorticoid receptor agonist, in rabbit models of ocular disease. Invest. Ophthalmol. Vis. Sci. 52 (3), 1422-1430. doi: 10.1167/iovs.10-5598

Sheppard, J. D., Comstock, T. L., and Cavet, M. E. (2016). Impact of the Topical Ophthalmic Corticosteroid Loteprednol Etabonate on Intraocular Pressure. Adv. Ther. 33 (4), 532-552. doi: 10.1007/s12325-016-0315-8

Stern, M. E., and Pflugfelder, S. C. (2004). Inflammation in dry eye. Ocul. Surf. 2 (2), 124-130. doi: 10.1016/S1542-0124(12)70148-9

Sullivan, B. D., Crews, L. A., Sönmez, B., De La Paz, M. F., Comert, E., Charoenrook, V., et al. (2012). Clinical utility of objective tests for dry eye disease: Variability over time and implications for clinical trials and disease management. Cornea. 31 (9), 1000-1008. doi: 10.1097/ICO.0b013e318242fd60

Tomlinson, A., Khanal, S., Ramaesh, K., Diaper, C., and McFadyen, A. (2006). Tear film osmolarity: Determination of a referent for dry eye diagnosis. Invest. Ophthalmol. Vis. Sci. 47(10), 4309-15 doi: 10.1167/iovs.05-1504
Wielders, L. H. P., Schouten, J. S. A. G., and Nuijts, R. M. M. A. (2018). Prevention of macular edema after cataract surgery. Curr. Opin. Ophthalmol. 29(1), 48-53. doi: 10.1097/ICU.0000000000000436

Zhang, N., Pan, H. F., and Ye, D. Q. (2011). Th22 in inflammatory and autoimmune disease: Prospects for therapeutic intervention. Mol. Cell. Biochem. 353 (1-2), 41-46. doi: 10.1007/s11010-011-0772-y

Conflict of Interest: The authors declare that the research was conducted in the absence of any commercial or financial relationships that could be construed as a potential conflict of interest.

Copyright (c) 2019 Bucolo, Fidilio, Fresta, Lazzara, Platania, Cantarella, Di Benedetto, Burgaletto, Bernardini, Piazza, Barabino and Drago. This is an openaccess article distributed under the terms of the Creative Commons Attribution License (CC BY). The use, distribution or reproduction in other forums is permitted, provided the original author(s) and the copyright owner(s) are credited and that the original publication in this journal is cited, in accordance with accepted academic practice. No use, distribution or reproduction is permitted which does not comply with these terms. 